\title{
What's New in Decoding Breast Cancer
}

\author{
Femke de Snoo \\ Medical Affairs, Agendia, Amsterdam, The Netherlands
}

\section{Introduction}

It has become apparent that breast cancer is a very complex disease that can affect individuals with seemingly identical clinicopathologic parameters very differently. For this reason, molecular classification of breast cancer is becoming increasingly important in determining treatment strategies for individual patients.

By using gene expression profiling on a genome-wide level for breast cancer, one can distinguish different molecular subtypes that have a similar biological behaviour. These subtypes are designated as luminal A, luminal B, basal and ERRB2. Even though these subtypes were developed without consideration of patient outcome, there are striking differences in aggressiveness between subtypes. Prognosis for patients with luminal A subtype is significantly better than for patients that have the basallike and ERBB2 subtypes, for which the survival times are shorter. The substratification of the luminal group into A and B subgroups has important prognostic implications; however, for determining the need for chemotherapy, more accepted characteristics for therapeutic decision-making - including risk stratification by multigene assays (gene expression signatures) - are preferred.

\section{Molecular Classification for Better Risk Stratification}

Risk stratification for therapeutic decision-making has historically been based on clinical and pathologic parameters such as patient age, tumor size, lymph node in- volvement, histological grade, estrogen/progesterone receptors (ER/PR), Ki-67, P53 and epidermal growth factor receptor type 2 (HER2) status. However, the NCCN and St. Gallen Guidelines have recently included molecular classification based on gene expression profiling in their decision trees. The two molecularly based tests that are currently included in these guidelines (Oncotype DX and MammaPrint) are designed to classify patients according to their risk of developing distant metastases and, hence, serve as a guide for treatment decisions. Both tests have a role in the contemporary management of breast cancer, and are utilized to help determine which patients would potentially benefit from adjuvant chemotherapy and/or hormonal therapy. The 21-gene Recurrence Score test determines whether patients have a high, intermediate or low risk of recurrence. The test was developed using RTPCR and measures the expression of 21 genes, 5 of which serve as controls. The 70-gene MammaPrint molecular classifier determines which patients have a high likelihood of developing distant metastases and which patients have a low risk. This test is performed on a gene expression microarray platform, measuring thousands of additional genes, for quality assurance/quality control, as well as for enabling read-out of additional molecular profiles.

\section{Predictors of Chemosensitivity}

The prognostic tools described herein allow for better estimation of risk of relapse; however, to determine whether this risk assessment also predicts treatment sen-

\section{KARGER}

Fax +41613061234 E-Mail karger@karger.ch www.karger.com

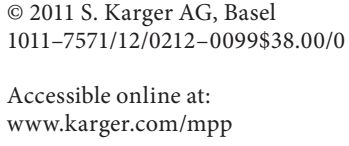

Accessible online at:

www.karger.com/mpp

Femke de Snoo

Agendia

Science Park 406

NL-1098 XH Amsterdam (The Netherlands)

E-Mail femke.desnoo@agendia.com 
sitivity, the MammaPrint profile has been analyzed in the neo-adjuvant setting [1]. This study was performed in 167 patients with stage II-III disease who received neo-adjuvant chemotherapy. The MammaPrint profile classified $144(86 \%)$ patients as 'high risk', of whom $29(20 \%)$ had a pathologic complete response to treatment, whereas none of the 'low risk' patients experienced a pathologic complete response. Notably, a MammaPrint 'high risk' result remained predictive of chemotherapy response when only ER+ HER2- patients were included in the analysis.

\section{I-SPY II Trial}

The ability of MammaPrint to identify patients who have the highest likelihood of responding to chemotherapy has initiated the adaptive I-SPY II (Investigation of Serial Studies to Predict Your Therapeutic Response with Imaging and Molecular Analysis 2) trial. This trial is intended to accelerate the identification of effective drugs for locally advanced breast cancer [2]. The trial started in 2010 and is intended to enrol 800 patients. Those patients who have a high-risk MammaPrint result measured on a core needle biopsy in the neoadjuvant setting will be randomized to receive standard therapy with or without an investigational cancer compound. This trial allows for multiple compounds to be tested simultaneously, and those with the highest response to be moved to the phase III setting. This process will both accelerate the drug evaluation process, as well as reduce the costs to pharma.

Trials like I-SPY II that evaluate drugs in molecularly based subgroups are initiated to address the concern that certain drugs may only be effective for patients with particular subtypes of breast cancer. The outcome of these trials and future implementation of this knowledge may improve the clinical management of breast cancer pa- tients by enabling the physician to decide who is most likely to benefit from which chemotherapy prior to surgery. Further, it may supplement information already available from validated multi-gene assays in helping the clinician decide on the best treatment for each patient.

\section{Molecular Classification in Daily Clinical Practice}

For the additional molecular risk classification provided by MammaPrint, the same tissue that is submitted for MammaPrint classification can then also be used to determine additional gene profiles, including TargetPrint, which determines the mRNA levels of ER, PR and HER2. It offers the opportunity for objective and more quantitative measurements, and can serve as an additional assessment of the tumor's hormonal receptor status. Another available diagnostic assay, BluePrint, is a molecular subtyping profile which determines the mRNA levels of 80 genes that best discriminate among the three distinct subtypes: basal type, luminal type and ERBB2 (HER2) type. Together with the clinicopathological criteria, MammaPrint and these additional genomic assays can be used to provide more personalized patient treatment decisions.

References

1 Straver ME, Glas AM, Hannemann J, Wesseling J, van de Vijver MJ, Rutgers EJ, Vrancken Peeters MJ, van Tinteren H, van't Veer LJ, Rodenhuis S: The 70-gene signature as a response predictor for neoadjuvant chemotherapy in breast cancer. Breast Cancer Res Treat 2010;119:551-558.

$\checkmark 2$ Barker AD, Sigman CC, Kelloff GJ, Hylton NM, Berry DA, Esserman LJ: I-SPY 2: an adaptive breast cancer trial design in the setting of neoadjuvant chemotherapy. Clin Pharmacol Ther 2009;86:97-100. 